\title{
Structural model splicing method based on well seismic combination
}

\author{
Dan Sun \\ Geological Research Institute of the Fourth Oil Production Plant of Daqing Oilfield Co. Daqing, Heilongjiang 163511 China
}

\begin{abstract}
The use of small blocks with model-building results for overall structural model splicing can unify fault understanding, effectively integrate existing small block model results, and form a unified and continuous overall model. This article focuses on the structural models of two small blocks in the central and western parts of a certain area, and studies the method of splicing structural models at the sedimentary unit level to lay the foundation for the precise tapping of the potential of the fault edge.
\end{abstract}

Keywords: Fine modeling of Fault; Construction model splicing.

\section{Introduction}

At present, an oilfield has entered into an extraordinarily high water-bearing period, the remaining oil distribution is scattered, and the edge of the fault has become a favorable area for residual oil tapping. Special well designs such as high-angle wells are designed to be close to the fault, so it is important to finely characterize the three-dimensional spatial spread of the fault and pinpoint the spatial location of the fault. Since the well-seismic combination of tectonic studies was carried out in an oilfield in 2007, it has deepened the understanding of tectonic features in three-dimensional space, achieved full coverage of well-seismic combination in the whole area, and finely delineated the sedimentary unit-level stratigraphic model, with a total of 18 three-dimensional fine tectonic models for small blocks. The drawbacks of the sub-block tectonic model have been found in practice in two main areas: firstly, the incomplete data of the fault model wells at the boundaries do not provide highly accurate cross-block model results, which limits the application of the results; secondly, the irregular shape of the generated fault polygons does not provide high accuracy in the delineation of the upper and lower plates. The well seismic based tectonic model splicing technique solves the drawbacks of the block tectonic model in the articulation area, and the seamless splicing of the tectonic model deepens the understanding of the overall tectonic features and plays an important role in the overall dredging work of the fault margin.

\section{Research ideas and advantages of constructing model splices}

\subsection{Research ideas for constructing model splices}

Tectonic model stitching requires a combination of information to accurately describe all geological formations within a range. The small block models are stored as multiple independent model files and only a single file can be manipulated and it is not possible to splice two model files. The traditional method of constructing a structural model is point- $>$ line- $>$ surface->body, the process of constructing a model is a reverse process from body- $>$ surface- $>$ line->point, first the results of the existing cell block structural model, into the database, the database for stitching fusion, and then the overall construction of the model. The splicing of structural models is a process of turning the parts into a whole. It requires a certain understanding of the structure in each small block. The modeling idea is based on the integration and unified management of the structural modeling data (Figure 1). The main stages can be divided into the following 3 phases: (1) Establishing the overall model work area. (2) Fusing the fault model results of each sub-block to establish the overall fault model. (3) Fusing the results of the layer model of each sub-block to establish the overall layer model. 


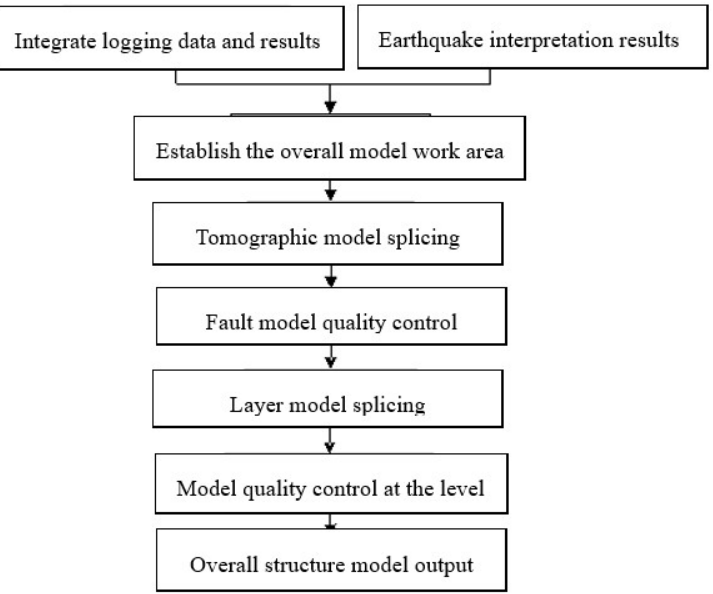

Figure 1 Constructing the model stitching process

\subsection{The advantages of constructing model splices}

Small block model stitching is a process of fusing data from zero to whole, with quality checks on well data combined with seismic data, which modelers can stitch to suit their needs and the computing power of their computers. This way the accuracy of the model is not compromised and the efficiency of building models of large areas is increased. Advantages of small block model stitching:

(1) High modeling efficiency. Because the small block model has been adjusted, it is only necessary to process the overlapping parts of multiple blocks for fine-tuning. The adjustment effort is only one third of that of conventional modelling and the modelling time is one fifth of that of conventional modelling.

(2) Improve the accuracy of boundary tectonics. Both the fault model and the stratigraphic model are derived from the original sub-block model. For the understanding of inter-block boundary tectonics, the well data and seismic interpretation results can be used during the splicing process to establish an overall unified 3D fine tectonic model to further understand the overall tectonic features.

\section{Key techniques for constructing model splices}

\subsection{Fault model stitching and fusion technology}

\subsubsection{Fault model data volume fusion}

The fault model data volume is extracted from the small block structural model, and the simulation is re-simulated under the control of the well break point data, and the fault is re-understood to ensure that the accuracy of the processed fault model is consistent with the original model. The fusion of the fault model data volume is divided into three categories: i. Inherited: the fault within the work area, extracting the fault model data and applying it directly to the fault model; ii. Reconstructed: the fault as a whole is at the boundary of two sub-blocks, using well seismic combined with the data to recalculate the fault model; iii. Spliced: the same fault is divided between different sub-blocks, extracting the fault model data between each sub-block separately. (see Figure 2)

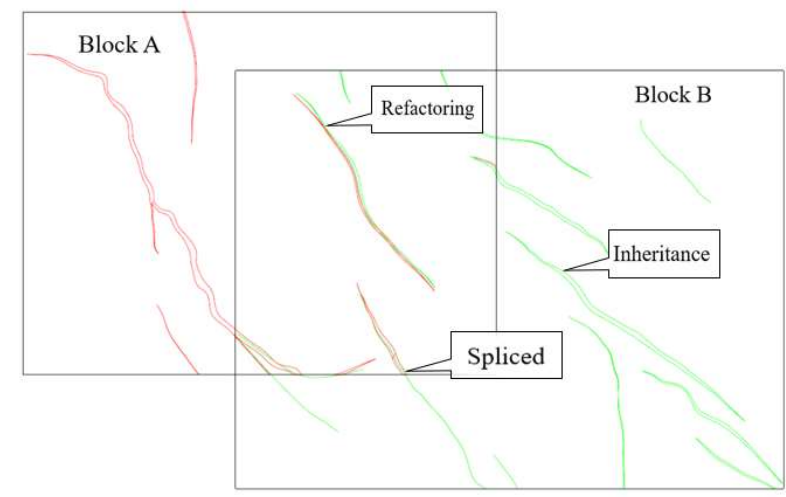

Figure 2 Schematic diagram of the fault splicing of two cell blocks

\subsubsection{Handling of fault intersection relations}

The conventional method for handling fault intersection relations is manual and manual adjustment, which is very laborious and time consuming. The fault model uses an "improved binary tree algorithm" when dealing with fault intersection relations, which is a new algorithm different from the traditional software fault modelling algorithm (pillar-based method Istrict binary tree method). This method uses the division of the fault line to define the range of the fault, avoiding the influence of modeling methods based on pillar and fault upper and lower walls. One-click adjustment of the cut-off relationship between faults can automatically handle the intersection of faults and faults, making editing fast and convenient. It not only improves the efficiency of fault modeling, but also improves the accuracy of fault model (Figure 3).

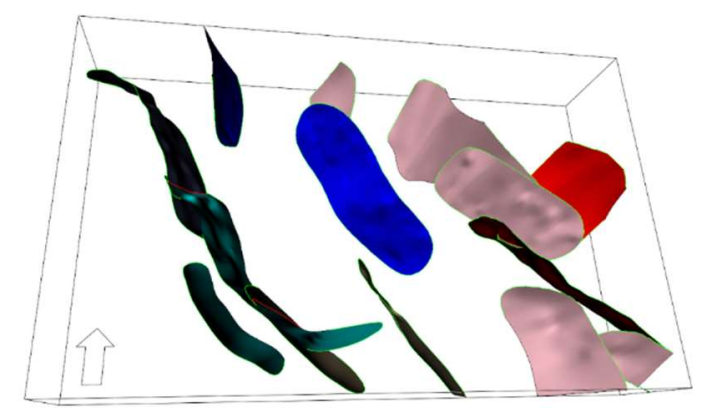

a Fault cross-cutting relationship before processing 


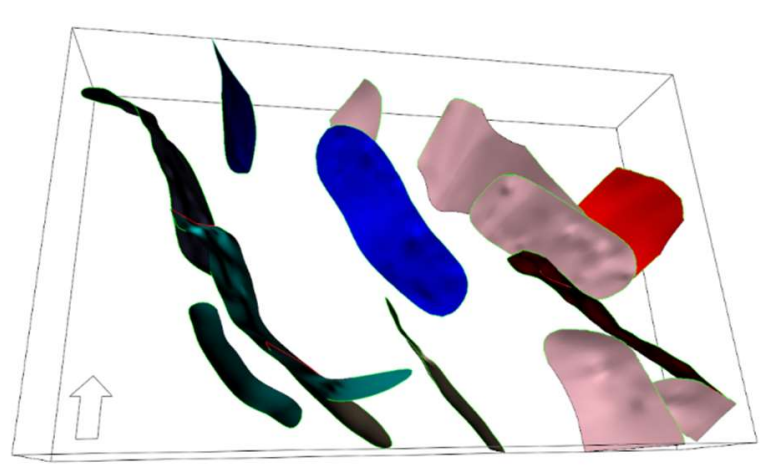

b Fault cross-cutting relationship after processing

Figure 3 Effect picture before and after processing of fault intersection relationship

\subsection{Layer model splicing and fusion technology}

The layer model is to establish a set of skeletal grid system within a given closed modeling boundary after the fault is accurately established. Therefore, when the structure changes at the boundary of the small block, the local layer model reconstruction method is used to reconstruct the horizon model of the overlap area: Using the boundary fault as the boundary, filter out the layer model data of 12 well spacing on each side of the fault (e.g. Figure 4 delete the data in the red border of block $\mathrm{A}$ and delete the data in the green border of block B), merge the layer model data of the two blocks into a new layer data, complete the layer model construction, complete the stitching of the layer model and unify the construction understanding.

In this paper, a hierarchical structure modelling technique is used to build a layer model, i.e. a layer model with seismic interpretation data (top surface model of the formation group) is built first, and the top surface model of each formation group is built using the drilling stratification data and the fused layer model data, and under the constraints of this model, the model of small layers or sedimentary units is built. Since the second-level structure is completed on the basis of the upper-level structure, the structure plane data except the overlap area is inherited from the small block model, so the hierarchical structure modeling can reduce the workload of small-level structure adjustment, and can Keep the structure trend consistent from layer to layer.
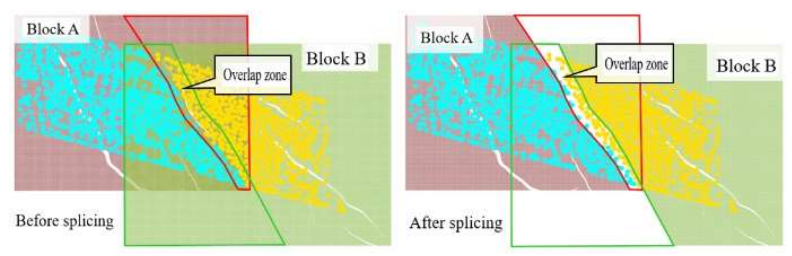

Figure 4 Schematic diagram of the splicing of the horizon model of the two small blocks

\section{Applications}

In order to effectively use the results of the block well seismic modelling, a three-dimensional tectonic model based on the combination of wells and seismic was developed for the central and western part of a district that already had the results of the block tectonic model, with a total of 1,583 wells (648 wells in the central part of a district and 935 wells in a district) in the central and western part of a district with a seismic work area of $16 \mathrm{~km} 2$ and 8 faults in the Saputo high objective. During the model splicing process, the breakpoint combination rate was $95.5 \%$, and the error of the tectonic surface was less than 2\%o (Figure 5). After the splicing process, the faults and layers of the tectonic model are spatially unified, and the results of the existing block model are effectively integrated to form a unified and continuous overall model, which improves the accuracy of the identification of boundary faults.

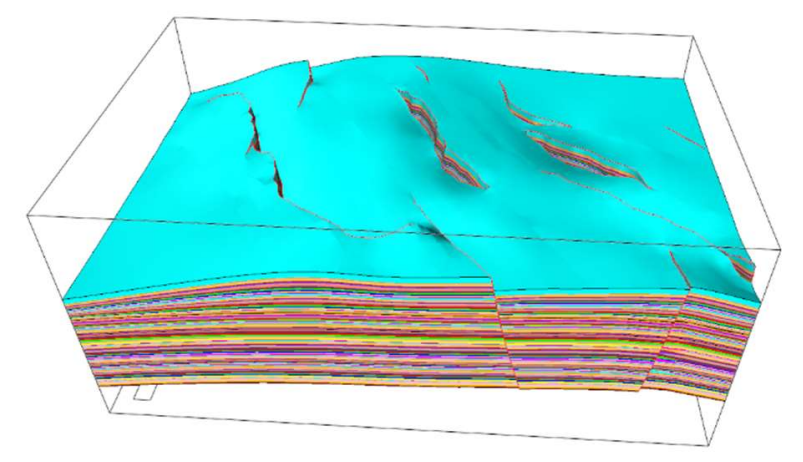

Figure. 5 Results of tectonic model splicing in the west-central part of a district

\section{Conclusion}

(1) The stitching technology of the construction model unifies the boundary fault recognition, effectively integrates the results of the existing cell block model, inherits the model data of the cell block, greatly reduces the model adjustment work, improves the efficiency of modeling, and saves a lot of time for the subsequent attribute modeling and tapping work.

(2) The establishment of the overall tectonic model can output the tectonic map of any layer, and any tectonic profile, and the continuous and consistent spreading of tectonic and fault features in three-dimensional space over a large area, which lays the foundation for the study of the overall tectonics and evolution of the whole region and the local fine tectonic features, and will greatly improve the effectiveness and efficiency of the application of tectonic research results. 


\section{References}

1. Jiang Yan, Li Jie, et al. Well Seismic Combined Integral Tectonic Modeling Techniques in the Daqing Changyuan Saltu Oilfield. Daqing Petroleum Geology and Development, 2015,34(1): 151-156.

2. Liu Lei, Jin Li, et al. Research Methods for Developing Seismic Tectonic Modeling Techniques. Highland Earthquakes, 2009, 21(3):46-51.

3. Zhang Yang, Wang Qi, et al. Tectonic Modeling and Applications for Complex Fracture Block Reservoirs. Liaoning Chemical Industry, 2012, 4(5):512-514.

4. Kong Hong. Application of RMS geological modeling software in MTZ area. Journal of Jianghan Petroleum Institute, 2003,25:9-10

5. Chong Renjie, Yu Xinghe. Key Points for Quality Control of 3D Geological Modeling of Reservoirs. Frontiers in Marine Geology, 2011,27(7):64-69. 